\title{
Article \\ The Fusion Gene Landscape in Taiwanese Patients with Non-Small Cell Lung Cancer
}

\author{
Ya-Sian Chang ${ }^{1,2,3,4}$, Siang-Jyun Tu ${ }^{2}$, Ju-Chen Yen ${ }^{1}$, Ya-Ting Lee ${ }^{1}$, Hsin-Yuan Fang 5 \\ and Jan-Gowth Chang 1,2,3,6,7,*(D)
}

1 Epigenome Research Center, China Medical University Hospital, Taichung 404332, Taiwan; t25074@mail.cmuh.org.tw (Y.-S.C.); t24399@mail.cmuh.org.tw (J.-C.Y.); t23701@mail.cmuh.org.tw (Y.-T.L.)

2 Department of Laboratory Medicine, China Medical University Hospital, Taichung 404332, Taiwan; t34752@mail.cmuh.org.tw

3 Center for Precision Medicine, China Medical University Hospital, Taichung 404332, Taiwan

4 Department of Medical Laboratory Science and Biotechnology, China Medical University, Taichung 404333, Taiwan

5 Department of Thoracic Surgery, China Medical University Hospital, Taichung 404332, Taiwan; d17573@mail.cmuh.org.tw

6 School of Medicine, China Medical University, Taichung 404333, Taiwan

7 Department of Bioinformatics and Medical Engineering, Asia University, Taichung 41354, Taiwan

* Correspondence: d6781@mail.cmuh.org.tw; Tel.: +886-4-22052121

check for updates

Citation: Chang, Y.-S.; Tu, S.-J.; Yen, J.-C.; Lee, Y.-T.; Fang, H.-Y.; Chang, J.-G. The Fusion Gene Landscape in Taiwanese Patients with Non-Small Cell Lung Cancer. Cancers 2021, 13, 1343. https://doi.org/10.3390/ cancers13061343

Academic Editor: Gennaro Ciliberto

Received: 21 February 2021

Accepted: 14 March 2021

Published: 16 March 2021

Publisher's Note: MDPI stays neutral with regard to jurisdictional claims in published maps and institutional affiliations.

Copyright: (c) 2021 by the authors. Licensee MDPI, Basel, Switzerland. This article is an open access article distributed under the terms and conditions of the Creative Commons Attribution (CC BY) license (https:/ / creativecommons.org/licenses/by/ $4.0 /)$.
Simple Summary: Human cancer genomes show a variety of alterations, such as single base changes, deletions, insertions, copy number changes, and gene fusions. Analyzing fusion gene transcripts may yield a novel and effective approach for selecting cancer treatments. However, few comprehensive analyses of gene fusions in non-small cell lung cancer (NSCLC) patients have been performed. Here, we characterized the fusion gene landscape of NSCLC in a case study of Taiwanese lung cancer patients. We concluded that some fusion genes likely play driver roles in carcinogenesis, while others act as passengers. We demonstrated that by using RNA-sequencing to detect gene fusion events, putative therapeutic drug targets could be identified, potentially leading to more precise therapies for NSCLC.

Abstract: Background: Analyzing fusion gene transcripts may yield an effective approach for selecting cancer treatments. However, few comprehensive analyses of fusions in non-small cell lung cancer (NSCLC) patients have been performed. Methods: We enrolled 54 patients with NSCLC, and performed RNA-sequencing (RNA-Seq). STAR (Spliced Transcripts Alignment to a Reference)-Fusion was used to identify fusions. Results: Of the 218 fusions discovered, 24 had been reported and the rest were novel. Three fusions had the highest occurrence rates. After integrating our gene expression and fusion data, we found that samples harboring fusions containing ASXL1, CACNA1A, EEF1A1, and RET also exhibited increased expression of these genes. We then searched for mutations and fusions in cancer driver genes in each sample and found that nine patients carried both mutations and fusions in cancer driver genes. Furthermore, we found a trend for mutual exclusivity between gene fusions and mutations in the same gene, with the exception of DMD, and we found that EGFR mutations are associated with the number of fusion genes. Finally, we identified kinase gene fusions, and potentially druggable fusions, which may play roles in lung cancer therapy. Conclusion: The clinical use of RNA-Seq for detecting driver fusion genes may play an important role in the treatment of lung cancer.

Keywords: NSCLC; RNA-sequencing; fusion genes; kinase; druggable

\section{Introduction}

Lung cancer is the most common malignant neoplasm in humans and the leading cause of cancer mortality worldwide [1]. Tobacco exposure is the number one risk factor for 
lung cancer [2]. Lung cancer is classified into different histological subtypes: non-small cell lung cancer (NSCLC) and small cell lung cancer. NSCLC accounts for $85 \%$ of all lung cancer diagnoses [3,4]. NSCLC has different characteristics in different ethnic groups; for example, the mutation frequencies of EGFR, KRAS, BRAF, and TP53 differ significantly between Asian and Western populations [5], which may result in different therapeutic outcomes.

Promising molecular-targeted therapies have been developed for the treatment of lung cancer. Therapies that are currently on the market include epidermal growth factor receptor (EGFR), tyrosine kinase inhibitors (gefitinib (Iressa ${ }^{\circledR}$ ), erlotinib (Tarceva ${ }^{\circledR}$ ), afatinib $\left(\right.$ Gilotrif $\left.^{\circledR}\right)$, and osimertinib (Tagrisso $\left.{ }^{\circledR}\right)$ ), anaplastic lymphoma kinase (ALK) inhibitors (crizotinib $\left(\right.$ Xalkori $\left.^{\circledR}\right)$, ceritinib (Zykadia $\left.{ }^{\circledR}\right)$, and alectinib (Alecensa $\left.{ }^{\circledR}\right)$ ), vascular endothelial growth factor-targeted monoclonal antibodies (bevacizumab (Avastin ${ }^{\circledR}$ ) and ramucirumab $\left(\right.$ Cyramza $\left.^{\circledR}\right)$ ), and anti-PD-1/anti-PD-L1 antibodies (pembrolizumab (Keytruda ${ }^{\circledR}$ ), nivolumab $\left(\right.$ Opdivo $\left.^{\circledR}\right)$, atezolizumab $\left(\right.$ Tecentriq $\left.^{\circledR}\right)$, and durvalumab (Imfinzi $\left.{ }^{\circledR}\right)$ ) [6]. However, resistance to cancer therapies remains a major clinical problem and involves all models of treatment, including molecular-targeted therapy and immunotherapy.

Detecting changes in human cancer genomes is crucial for developing targeted therapies for precision medicine. Most recent studies have focused on detecting mutations, insertions, deletions, and copy number changes when examining cancer genomes; broadening the search to include fusion transcripts may better aid cancer treatment selection. The prevalence of gene fusions in human cancers is approximately $20 \%$ [7]. However, the frequency of gene fusions varies greatly among cancer types, and most gene fusions are tumor specific, which supports the need for different oncogenic and therapeutic approaches. Examples of common gene fusions in cancer include EWSR1-FLI1 in Ewing's sarcoma [8], $B C R-A B L 1$ in chronic myeloid leukemia [9], and DNAJB1-PRKACA in fibrolamellar carcinoma [10]. Of the 28 Food and Drug Administration-approved drugs, 11 were approved to target fusion genes ( $A L K, R O S 1, N T R K, P M L-R A R A$ and $B C R-A B L), 16$ were approved to target non-fusion genes (EGFR, BRAF, BRCA, HER2, FLT3, IDH1, and IDH2), and one was approved to target both fusion and non-fusion genes (the fusion target $B C R-A B L$ and the non-fusion target KIT) [11]. Fusion genes are therefore promising targets for the treatment of cancer.

The accurate detection of gene fusions in patients has the potential to improve patient care. Multiple methods have been developed to detect gene fusions, such as fluorescence in situ hybridization, quantitative real-time polymerase chain reaction, G-banding cytogenetics, and immunohistochemistry. These methods are time-consuming and labor intensive and can detect only one or several known fusion transcripts. Advancements in next-generation sequencing technology have enabled the rapid assessment of many potential gene fusions in a single test, including known and uncharacterized gene fusions via RNA-Sequencing (RNA-Seq), which is currently the most common method for analyzing multiple fusion genes on a genomic scale.

Previous studies typically used The Cancer Genome Atlas (TCGA) RNA-Seq database to analyze fusions in different tumor types [12-14], rarely using their own data. Rudin et al. [15] performed the first genome-wide comprehensive analysis of gene fusions in lung cancer patients using their own case study. They identified multiple fusion transcripts and a recurrent fusion of RLF-MYCL1. Subsequently, Sao et al. found 45 fusion genes in 87 lung cancer patients, 8 of which were chimeric tyrosine kinase genes (EML4-ALK, KIF5B-RET, CD74-ROS1, SLC34A2-ROS1, CCDC6-ROS1, SCAF11-PDGFRA, FGFR2-CIT, and AXL$M B I P)$, which play important roles in cancer development [16]. In another report, the gene fusions of six never-smoking female patients with lung cancer were analyzed by RNA-Seq; these patients had no mutations in KRAS or EGFR and were negative for EML4-ALK [17]. Recently, Miyanaga et al. identified eight fusion genes in six pulmonary carcinoid patients using RNA-Seq [18].

In this study, we performed RNA-Seq to identify fusion events in a population of Taiwanese patients with NSCLC. Moreover, we investigated the relationship between the fusion status and gene expression/mutation status of genes. 


\section{Results}

\subsection{Identification of Fusion Genes in NSCLC}

We performed RNA-Seq in samples resected from 54 NSCLC patients (54 tumor tissues and 8 matched normal tissues) and applied a screening process to identify fusion genes in NSCLC (Figure 1). Using STAR-Fusion, we identified 311 fusion genes. Next, we filtered reads using additional criteria: excluding fusions that were expressed in normal tissues, number of reads $>10$, and reads with an intrachromosomal rearrangement distance cutoff $>10 \mathrm{~kb}$. The fusion types included in the search were fusions between two protein coding genes, between two long non-coding RNAs (lncRNAs), of a protein coding gene followed by an lncRNA, and of an lncRNA followed by a protein coding gene. This study identified 218 fusion genes from 49 samples (Table S1); 24 of these fusions have been reported: AC010998.1-WDR11-AS1, ACOT9-APOO, AHNAK-NXF1, AL135923.2-DMAC1, AL445647.1MIR4500HG, CASC19-CASC8, FANCA-FP236383.1, FGFR3-TACC3, FRAS1-MRPL1, FTH1FTL, JMJD1C-AS1-REEP3, KIF5B-RET, KRT5-KRT17, LINC02159-ATP10B, MTMR3-UQCR10, MUC4-TNK2, PSAP-SFTPC, RAB31-PPP4R1-AS1, SBNO1-RILPL1, SLC12A2-CCDC192, TBC1D15-RAB21, TMEM123-YAP1, TPH2-TRHDE, and UBAP2-DCAF12. These 24 known gene fusions were present in 17 of our NSCLC patients (Tables S2 and S3). We validated 77 novel fusion genes using reverse transcription-polymerase chain reaction (RT-PCR) and Sanger sequencing (Figure S1).

\section{Unfiltered fusions from STAR-Fusion:}

\section{1}

Without normal tissue fusions:

\section{9}

Fusion reads (junction reads plus spanning reads) $>10$ :

\section{0}

Intrachromosomal rearrangement distance cutoff $>10 \mathrm{~kb}$ :

\section{5}

Fusion gene types focus on protein coding-protein coding, lncRNA-lncRNA, protein coding-lncRNA, lncRNA-protein coding:

\section{8}

Figure 1. Overview of the filtering process of fusion gene RNA-Seq reads obtained from NSCLC (non-small cell lung cancer) samples.

To identify novel fusions that paly roles in cancer development, we selected transcripts predicted to encode protein products (108 fusion genes) and then applied the IUPred2A tool to predict protein functional changes. As a result, 25 fusion proteins were predicted to exhibit disordered functions (Figure S2) and were found in 15 of the NSCLC patients. We also calculated the number of important mutants in 299 known cancer driver genes (CDGs) [19] in each case. The average number of important variants in each case was approximately three. Nine patients had more than the average number of CDG variants, 
suggesting that the fusion genes in these patients may play a passenger role as traditional passenger variants. However, six patients, in whom seven novel gene fusions (NUP153DHX16, PFDN4-ASXL1, PPP2R5A-NENF, RBM19-BRAP, HTT-CDH12, SFTPA1-FTL, and $P P P 1 R 37-T H R A$ ) were found, had fewer than average important CDG variants, suggesting that these seven fusion genes may play a driver role as traditional driver variants.

Three fusion genes exhibited particularly high occurrence rates (=number of patients with fusion genes/49): FTH1-FTL, ARHGAP21-PRTFDC1, and LINC02159-ATP10B. The occurrence rate was approximately $6.12 \%$ (3/49) for FTH1-FTL and 4.08\% (2/49) for the other two fusions. Other fusion genes were mutually exclusive. The FTH1-FTL and LINC02159$A T P 10 B$ fusions have previously been reported in the Cancer Cell Line Encyclopedia and TCGA datasets; however, their functions have not been well characterized.

\subsection{The Clinical Significance of Fusion Genes Involving Oncogenes or TSGs}

Next, we grouped the fusion genes into those involving oncogenes or TSGs and then determined which, if any, of the fusion genes were expression outliers. Five of the fusion genes involved a TSG (PFDN4-ASXL1, BCOR-DMD, CDK12-PNMT, CSDE1-TERT, and KANSL1-AC091132.4) and were found in five patients. The TSG ASXL1 was the only observed expression outlier, i.e., ASXL1 was aberrantly expressed in the samples harboring ASXL1 fusion genes (Figure 2A and Figure S3A-D). In five patients, seven fusion genes involved an oncogene (FGFR3-TACC3, EEF1A1-RPL3, DMD-PHEX, ACOT9-DMD, BCOR$D M D, D H X 34-C A C N A 1 A$, and KIF5B-RET). Three of these oncogenes (CACNA1A, EEF1A1, and RET) were classified as expression outliers (Figure 2B-D and Figure S3E,F).

(A)

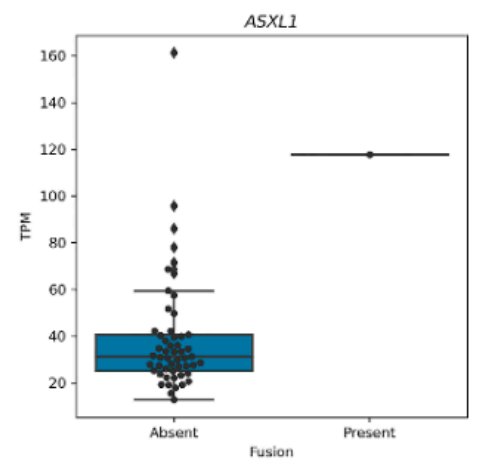

(C)

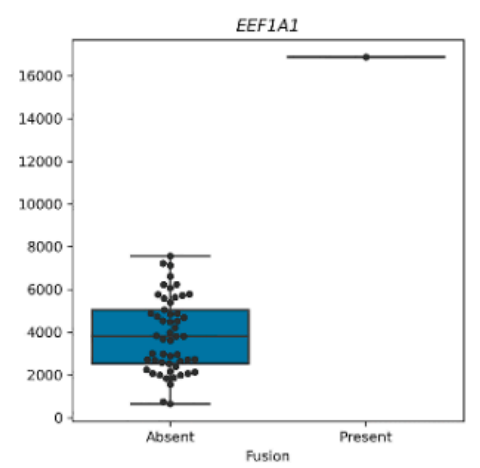

(B)

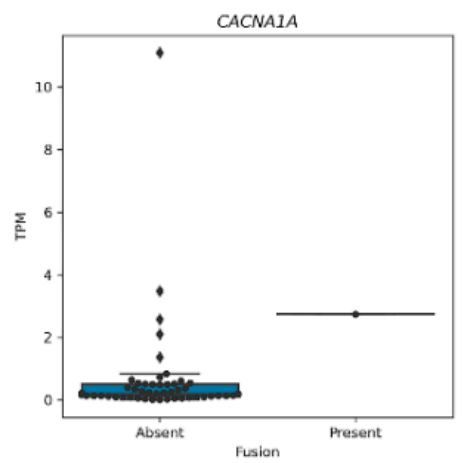

(D)

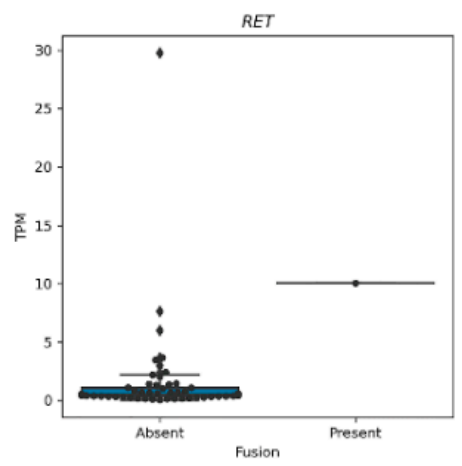

Figure 2. Expression levels of genes present in fusions; expression outliers are shown. (A) ASXL1 expression in samples with or without $A S X L 1$ fusion genes. (B) CACNA1A expression in samples with or without $C A C N A 1 A$ fusion genes. (C) EEF1A1 expression in samples with or without EEF1A1 fusion genes. (D) RET expression in samples with or without RET fusion genes. 
The clinicopathological features of the NSCLC patients with fusion genes involving TSGs (tumor suppressor genes) and/or oncogenes were investigated. We found that age and grade were correlated with the presence of fusion genes involving TSGs $(p=0.0208$ and $p=0.0363$, respectively), whereas grade was correlated with the presence of fusion genes involving oncogenes $(p=0.0086)$ (Table 1$)$. Patients with a higher grade have a higher relative percentage of fusion genes involving oncogenes and TSGs, but no correlation with tumor stage. No significant correlation was found between overall survival and the presence of fusion genes involving TSGs or oncogenes (Figure S4A,B).

Table 1. Relationships between fusion genes detected using RNA-Seq and clinicopathological characteristics in 54 patients with NSCLC.

\begin{tabular}{|c|c|c|c|c|c|c|c|c|c|}
\hline \multirow[t]{2}{*}{ Features } & & \multicolumn{3}{|c|}{$\begin{array}{c}\text { Fusion Genes } \\
\text { Involving Oncogenes }\end{array}$} & \multirow[t]{2}{*}{$p$-Value } & \multicolumn{3}{|c|}{$\begin{array}{c}\text { Fusion Genes Involving } \\
\text { TSGs }\end{array}$} & \multirow[t]{2}{*}{$p$-Value } \\
\hline & & No & Yes & Total & & No & Yes & Total & \\
\hline \multirow[t]{2}{*}{ Gender } & Male & 26 & 2 & 28 & 0.6633 & 26 & 2 & 28 & 0.6633 \\
\hline & Female & 23 & 3 & 26 & & 23 & 3 & 26 & \\
\hline \multirow[t]{2}{*}{ Age } & $<70$ & 25 & 3 & 28 & 1.000 & 28 & 0 & 28 & 0.0208 \\
\hline & $\geq 70$ & 24 & 2 & 26 & & 21 & 5 & 26 & \\
\hline \multirow[t]{2}{*}{ Stage } & $\overline{\mathrm{I}}-\mathrm{II}$ & 34 & 2 & 36 & 0.1626 & 33 & 3 & 36 & 0.6374 \\
\hline & III-IV & 13 & 3 & 16 & & 14 & 2 & 16 & \\
\hline \multirow[t]{2}{*}{ Grade } & $1-2$ & 38 & 1 & 39 & 0.0086 & 38 & 1 & 39 & 0.0363 \\
\hline & $3-4$ & 8 & 4 & 12 & & 9 & 3 & 12 & \\
\hline \multirow[t]{2}{*}{ Histology type } & ADC & 41 & 3 & 44 & 0.2273 & 39 & 5 & 44 & 0.5707 \\
\hline & $\mathrm{SqCC}$ & 8 & 2 & 10 & & 10 & 0 & 10 & \\
\hline \multirow[t]{2}{*}{ Smoking status } & Non-smokers & 32 & 3 & 35 & 1.000 & 31 & 4 & 35 & 0.6461 \\
\hline & Smokers & 17 & 2 & 19 & & 18 & 1 & 17 & \\
\hline
\end{tabular}

p-value by Fisher's exact test. All subjects underwent surgical resection. Patients were diagnosed according to the 2015 World Health Organization and International Association for the Study of Lung Cancer guidelines. Staging was based on the guidelines of the eighth edition of the TNM classification for NSCLC.

\subsection{Mutual Exclusivity of Gene Fusions and Gene Mutations}

Mutations in oncogenes and TSGs play key roles in cancer induction, and fusions involving such genes are likely to contribute to cancer development. To better understand how fusion genes contribute to carcinogenesis in NSCLC patients, we systematically profiled mutations and fusions in 299 known CDGs (cancer driver genes) [19]. We then classified the patients into three groups (some overlapping): those with CDG mutations known to be pathogenic or likely to be pathogenic (according to the ClinVar database), those with CDG mutations with predicted pathogenicity, and those with a gene fusion involving a known CDG.

Our results showed that $77.78 \%(42 / 54)$ and $22.22 \%(12 / 54)$ of the patients carried known driver mutations and predicted pathogenic driver mutations, respectively (Table S4). Of the 42 patients with known driver mutations, seven carried both driver mutations and driver fusion events. Of the 12 patients with predicted pathogenic mutations only, two had both a fusion gene and a predicted pathogenic mutation (Figure 3).

Of the 35 patients with known driver mutations only, the total number of variants per sample ranged from 1 to 8 , averaging 3.91. The total number of variants per sample in the seven patients with known driver mutations and fusions ranged from 1 to 5 , with an average of 3.00. The samples with fusions carried fewer mutations compared with those without fusions, although this difference was not significant. In the 10 patients with predicted pathogenic mutations only, the total number of variants per sample ranged from 1 to 5 , with an average of 2.8 , which was lower than the average number recorded in the patients with known driver mutations. There was only one variant per sample in the two patients with predicted pathogenic mutations and fusions. Similar to the patients with known driver mutations, samples harboring fusions of predicted CDGs had fewer mutation variants. 


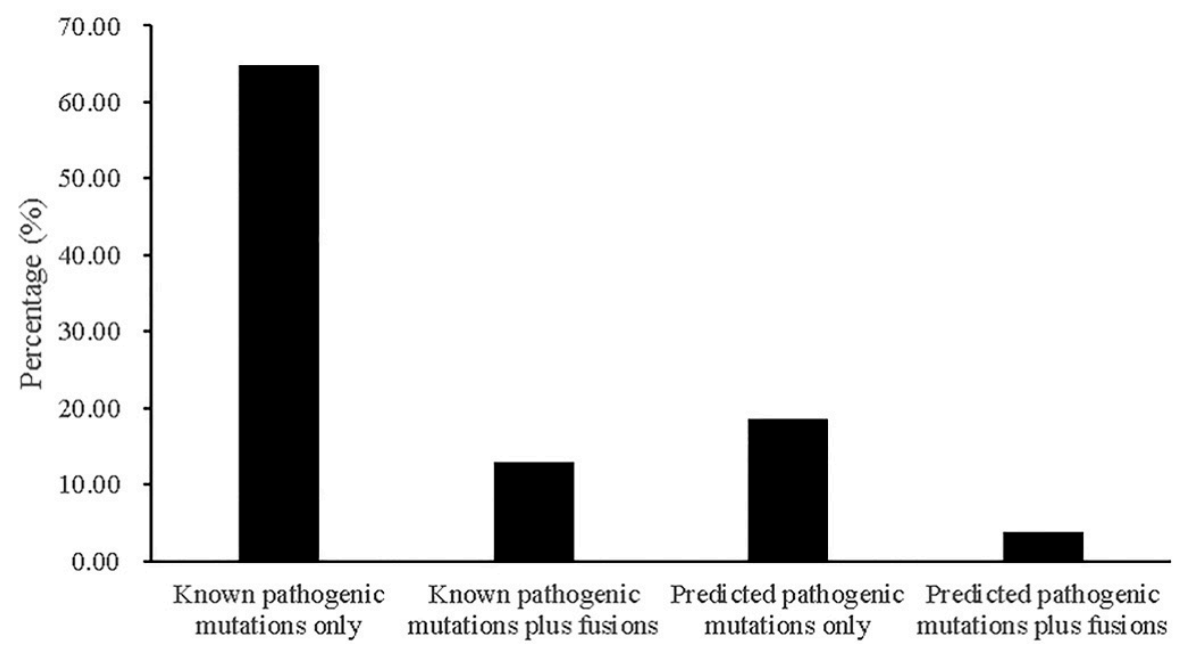

Figure 3. The chart shows the percentages of samples containing known pathogenic mutations only, predicted pathogenic mutations only, known pathogenic mutations plus fusions, or predicted pathogenic mutations plus fusions in 299 known cancer driver genes.

We then examined copy number variations in the 10 patients who carried predicted pathogenic mutations only. Seven of these patients had increased copy numbers of oncogenes and decreased copy numbers of TSGs (Table 2). These data suggest that the predicted pathogenic variants in the remaining three patients likely act as true driver mutations.

Table 2. Summary of gains of oncogenes and losses of TSGs (tumor suppressor genes) in seven NSCLC patients.

\begin{tabular}{ccc}
\hline Sample & Gains of Oncogenes & Losses of TSGs \\
\hline Lung-T151 & KIT, CEBPA, PLCB4 & ATM, KMT2A, MEN1, WT1, STK11 \\
Lung-T218 & DICER1, GNAS, DMD & none \\
Lung-T227 & SMARCA4 & none \\
Lung-T236 & KIT, RET, DICER1, GNAS, COL5A1 & none \\
Lung-T291 & $K I T, C A R D 11$, WT1, DICER1, MAX, CDH1, EP300 & none \\
Lung-T95 & $M S H 3$, PTPN11, DICER1, CDH1, EP300, MYH9 & BAP1, ATM, CHD8, LZTR1, CDKN2A \\
Lung-T430 & GNAS, MACF1 & n
\end{tabular}

We further examined the relationship between fusions and mutations in the same driver gene. Of the samples from eight patients, 10 harbored fusion genes that differed from the genes with mutations. Only one patient carried a fusion and mutation involving the same gene $(D M D)$. This suggests that the majority of gene fusions and mutations are mutually exclusive and do not tend to occur in the same gene.

\subsection{Correlation between EGFR Mutations and the Number of Fusion Genes}

EGFR mutations play important roles in the development and treatment of lung cancer, and they occur frequently in Taiwanese people and other Asians. The relationship between EGFR mutations and fusion genes has not been explored previously. We investigated whether fusion genes are correlated with EGFR mutations. The results showed that the EGFR mutations are not associated with driver fusions (Table 3). However, we found that EGFR mutations are associated with the number of fusion genes $(p=0.0038)$, and patients with EGFR mutations may have more fusion genes $(\geq 5)$ than patients with wildtype EGFR.

Table 3. Correlation between fusions and EGFR mutations.

\begin{tabular}{|c|c|c|c|c|c|c|c|c|c|}
\hline \multirow[t]{2}{*}{ Features } & \multirow[t]{2}{*}{ Genotype } & \multicolumn{3}{|c|}{ Fusion Genes Involving 299 CDGs } & \multirow[t]{2}{*}{$p$-Value } & \multicolumn{3}{|c|}{ Number of Fusion Genes } & \multirow[t]{2}{*}{$p$-Value } \\
\hline & & No & Yes & Total & & $<5$ & $\geq 5$ & Total & \\
\hline \multirow[t]{2}{*}{ EGFR } & Mutation & 22 & 5 & 27 & 1.000 & 12 & 15 & 27 & 0.0038 \\
\hline & Wildtype & 23 & 4 & 27 & & 23 & 4 & 27 & \\
\hline
\end{tabular}




\subsection{Structure and Spectrum of Kinase Gene Fusions}

In total, we detected 21 kinase gene fusions, of which 12 contained the kinase at the $5^{\prime}$ end (CAMK1D, CDK12, DGKZ, DMPK, DYRK4, FGFR3, MAGI1, MAST4, PTK2, RPS6KA1, SMG1, and TAOK1), and nine at the $3^{\prime}$ end (ERN2, GUCY2C, MAGI1, NIM1K, RET, STK10, TEK, TNK2, and WNK3). Analysis of the catalytic kinase domains using the PFAM domain database showed that six of the kinase gene fusions retained an intact kinase domain (CDK12, DMPK, ERN2, FGFR3, NIM1K, and SMG1). We then used AGFusion software to check whether the annotated kinase domain is still present in the fusion transcripts, allowing us to classify the fusions as those with an intact kinase domain versus a disrupted kinase domain. Three fusions (DMPK-SYMPK, FGFR3-TACC3, and SMG1ERN2) were in-frame, whereas CARMIL1-NIM1K and CDK12-PNMT were out-of-frame. Four of the fusions (CARMIL1-NIM1K, CDK12-PNMT, FGFR3-TACC3, and SMG1-ERN2) had intact kinase domains. The breakpoint position of five fusions was within the coding sequences (Figure 4).
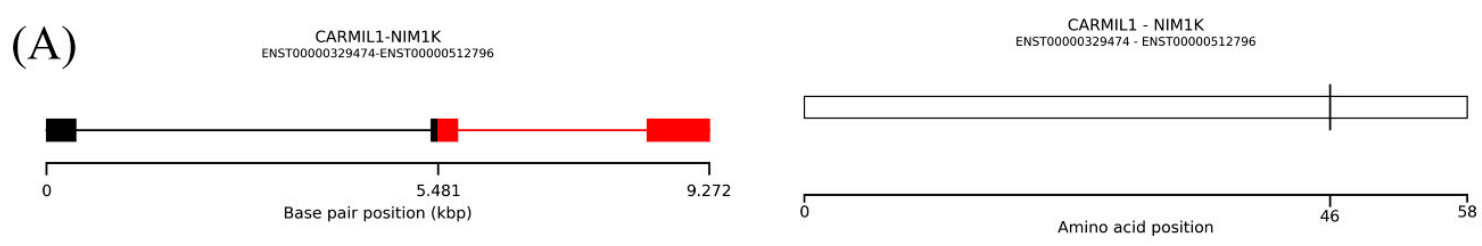

(B)
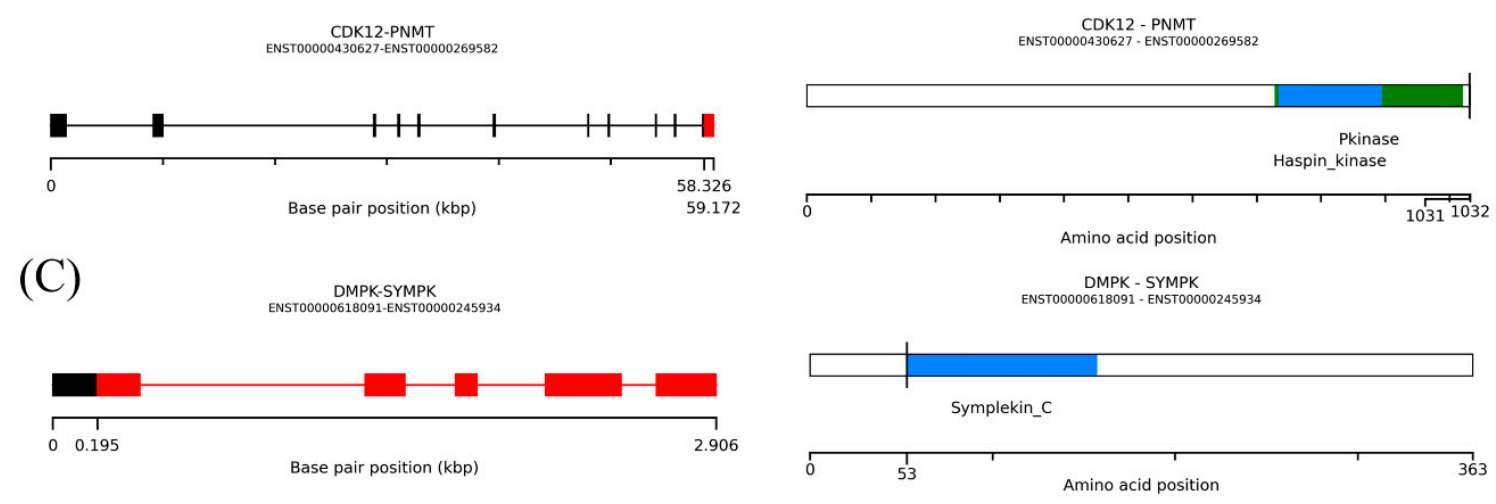

(D)
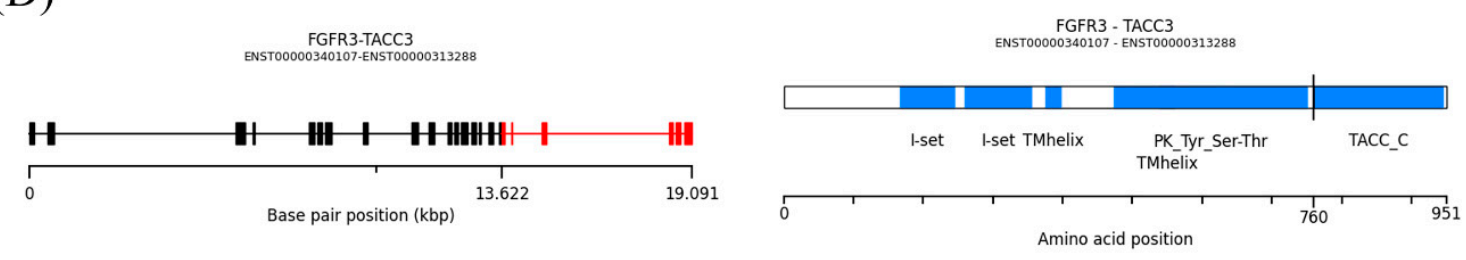

(E)

SMG1-ERN2
ENST00000446231-ENSTO0000256797
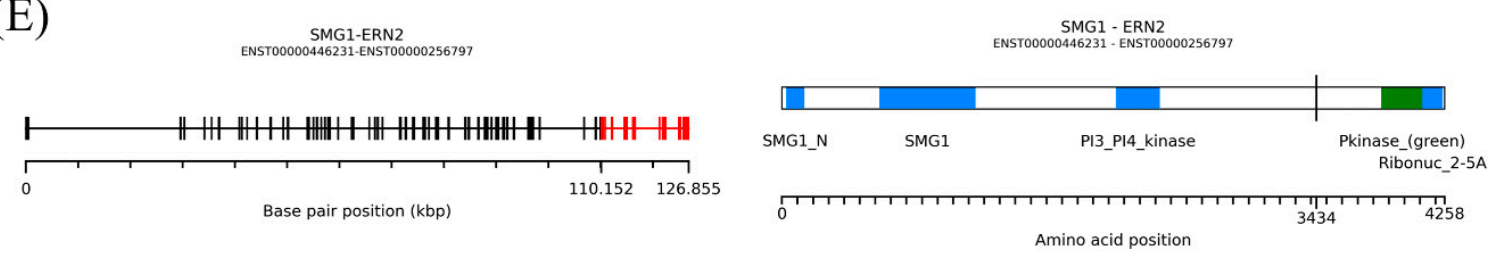

Figure 4. Predicted structures of kinase gene fusions. (A) CARMIL1-NIM1K, (B) CDK12-PNMT, (C) DMPK-SYMPK, (D) FGFR3-TACC3, and (E) SMG1-ERN2.

\subsection{Contributions of Fusions to Cancer Treatment}

Using the Database of Evidence for Precision Oncology, we identified clinically relevant genomic fusion alterations in five patients $(10.20 \%)$. Clinically relevant fusion alterations were found in the following genes: FGFR3 $(1,1.85 \%), N O T C H 2(2,3.70 \%)$, 
TMPRSS2 (1, 1.85\%), and RET (1, 1.85\%). The drugs for FGFR3, NOTCH2, and TMPRSS2 were off-label use, while RET was on-label use.

We also examined drug-related fusion genes using our RNA-Seq data, including $A L K$, ROS1, NTRK1/2, EGFR, BRAF, and RET, and only one RET fusion was found, which might have been due to the small sample size.

\section{Discussion}

Our study explored the clinical significance of fusion genes in lung cancer for the first time in Taiwan. We examined whether the concepts of driver and passenger fusions are useful in explaining the importance of fusion genes in the development of lung cancer, just as driver and passenger mutations. Therefore, we classified novel fusions as driver or passenger fusions according to the number of detected cancer driver gene variants. If a patient has fewer than the average number of gene variants and no known driver variants, any fusion gene observed is called a driver fusion. By contrast, if the patient has more than the average number of gene variants, especially known driver variants, any fusion gene observed is called a passenger fusion.

We found that $16.66 \%$ of the patients $(9 / 54)$ harbored fusions involving CDGs, together with known cancer driver mutations (seven patients) or predicted pathogenic variants (two patients). We then examined the combined oncogene mutations in nine samples and found mutations in PIK3CA, FGFR2, EGFR, DMD, PTPDC1, and DIAPH2 in patients who also harbored driver fusions. We therefore speculated that these genes are relatively weak oncogenes that require additional alterations for progression to carcinogenesis (Figure S5A). Moreover, no gene fusions involving CDGs were found in patients with mutations in the oncogenes CTNNB1, DACH1, ERBB2, FGFR3, GNAS, GTF2I, KLF5, KRAS, MSH3, and POLRMT. This suggests that these genes are relatively strong oncogenes that drive cancer development without the need for additional driver gene fusions (Figure S5B). Choi et al. reported the presence of somatic mutations in various TSGs in colorectal cancer patients who also carried fusion genes, whereas no non-synonymous somatic oncogene mutations were observed in patients harboring fusion genes [20]. In this study, we found that somatic mutations in various oncogenes and TSGs were present in NSCLC patients positive for fusion genes, although our results differed from those of Choi et al.; we propose that the strength of the oncogenic effects of genes should be considered. Some weak oncogenic variants require other oncogenes, TSGs, or fusion genes to drive cancer. In addition, Choi et al. did not find any somatic mutations within either the partner gene of a fusion [20]. Similarly, our results suggest that mutations are rarely present in fusion genes. Only one patient with a mutation in the DMD gene had a concomitant fusion involving $D M D$; this implies that the oncogenic effect of the $D M D$ mutation or fusion is weak when present alone.

The presence of fusion genes may result in altered expression of one or both of the fusion gene partners [21]. Samples harboring fusions containing TSGs (ASXL1) or oncogenes (CACNA1A, EEF1A1, and RET) exhibited increased expression of those genes relative to the samples without fusions. Gao et al. demonstrated that fusion genes influence oncogene expression, usually via overexpression of oncogenes and reduced expression of TSGs [7]. Further investigation is needed to determine whether the dysregulated oncogene expression is caused by the fusion events or another factor, as in the study by Lu et al. [22].

In hematopoietic malignancies and solid tumors, gene fusions can result in kinase activation. Kinases represent a major class of oncogenes, and two well-known examples of cancer-promoting kinase gene fusions are the $B C R-A B L 1$ fusion in leukemia patients and fusion of $A L K$ to various other proteins in lung carcinomas and mesenchymal tumors. Drugs that target these two kinases are now available [23,24]. In this study, we found 21 kinase gene fusions, which we translated into peptide sequences; five of these kinase gene fusions had intact functional catalytic domains (DMPK-SYMPK, FGFR3-TACC3, CARMIL1-NIM1K, CDK12-PNMT, and SMG1-ERN2). Our findings could provide a useful reference for cancer drug development. FGFR3-TACC3 is a common fusion in several 
cancer types [14]. Therefore, we propose FGFR3-TACC3 as a candidate target for drug development to treat NSCLC.

A correlation was observed for the grade and fusion genes involving TSGs and oncogenes but no correlation with tumor stage. Fusion genes involving TSGs and oncogenes in the tumors at higher grades were significantly more frequent than those in the tumors at lower grades. These results have also been found in prostate and brain tumors in the specific fusion genes TMPRSS2-ERG and ARHGEF2-NTRK1, which involved ERG and NTRK1 oncogenes $[25,26]$. We also explored the correlation between EGFR mutations and the number of fusion genes in each case. The results showed that cases with EGFR mutations had more fusion genes. The association between the occurrence of fusion genes and the EGFR genetic background may result from tumors with an EGFR mutation exhibiting increased cell proliferation and survival, which then increases the likelihood of fusion genes occurring [27].

This study has the following limitations. First, the cohort size was too small to make more definitive conclusions and obtain statistical significance in the correlation analysis; several gene fusions were found in three or fewer patients, making populationwise interpretation almost impossible. Second, we did not perform functional studies to confirm the effects of driver fusion genes, which might have influenced our subgrouping accuracy. Third, the study is based solely on the Taiwanese population. The results should be re-evaluated in other studies based on larger heterogeneous cohorts, to further validate our findings.

\section{Materials and Methods}

\subsection{Patient Samples}

Tissue specimens were obtained from 54 Taiwanese patients with lung cancer who had undergone surgical resection between May 2007 and April 2014 at the China Medical University Hospital. Of these patients, eight had matched tumor and normal tissues. The 54 lung tumors comprised 44 adenocarcinomas and 10 squamous cell carcinomas. The surgically resected specimens were immediately dissected and preserved in liquid nitrogen. The present study was approved by the Institutional Review Board of the China Medical University Hospital (CMUH106-REC1-053).

\subsection{RNA-Seq}

Total RNA was extracted from the clinical tissue samples using the NucleoSpin ${ }^{\circledR}$ RNA Kit (Macherey-Nagel, Düren, Germany) according to the manufacturer's instructions. The quality, quantity, and integrity of the total RNA were evaluated using a NanoDrop1000 spectrophotometer and Agilent 2100 Bioanalyzer (Agilent Technologies, Santa Clara, CA, USA). Samples with an RNA integrity number $>6.0$ were used for RNA-Seq. A barcoded mRNA library was generated using the TruSeq Stranded mRNA Library Preparation Kit (Illumina, San Diego, CA, USA). The libraries were sequenced on the Illumina Nova Seq 6000 (Illumina) using $2 \times 151$ bp paired-end sequencing flow cells, according to the manufacturer's instructions.

\subsection{RNA-Seq Data Analysis}

We used Trimmomatic for quality control of the RNA-Seq data [28], as described previously [29]. For gene expression, the reads that passed quality control were aligned with the GRCh38 human genome using HISAT2 [30], and featureCounts was used to quantify gene expression with annotation done using GENCODE v22 without mitochondrial genes [31]. The transcripts-per-million normalization method was applied.

To detect fusion transcripts, STAR-Fusion was applied using reads that passed quality control and the CTAT library downloaded from https: / / data.broadinstitute.org/Trinity/ CTAT_RESOURCE_LIB/_genome_libs_StarFv1.7/ (accessed on 17 August 2019). FusionInspector, a sub-module of STAR-Fusion, was used for IGV validation and annotation [32]. To remove false-positive fusions, fusions reported in eight tumor-adjacent 
normal tissues were filtered out. Furthermore, only fusion read counts $>10$ were included; the fusion read count was calculated by summing the "JunctionReadCount" and "SpanningFragCount" from the STAR-Fusion report. If a fusion rearrangement was present in the same chromosome, the distance between the left and right breakpoints had to be $>10$ kb. ChimerDB2, ChimerKB, ChimerPub, ChimerSeq, Cosmic, YOSHIHARA TCGA [33], Klijin CellLines [34], GUO2018CR TCGA [7], TumorFusionNAR2018 [13], TCGA StarF2019 (fusions found by STAR-Fusion v1.5.0 across corresponding TCGA datasets), and CCLE StarF2019 (fusions identified in the Cancer Cell Line Encyclopedia RNA-Seq datasets) were used to find reported gene fusions. Novel fusion protein disorders were detected using IUPred2A [35]. We used the PFAM domain database (https:// pfam.xfam.org/, accessed on 2 November 2020) to check for genes annotated with a kinase domain and AGFusion to examine fusion transcripts with an intact or disrupted kinase domain [36]. Fusion events and their respective drug therapies were found using the Database of Evidence for Precision Oncology [37].

\subsection{Reverse Transcription-Polymerase Chain Reaction and Sanger Sequencing}

Fusion genes were validated using RT-PCR with primers that target fusion gene breakpoints. Total RNA was extracted from the clinical tissue samples using the NucleoSpin ${ }^{\circledR}$ RNA Kit (Macherey-Nagel). RNA samples (2000 ng) were reverse-transcribed into cDNA using High-Capacity cDNA Reverse Transcription kits (Applied Biosystems, Foster City, CA, USA). The PCR primers are shown in Table S5. The PCR program consisted of $5 \mathrm{~min}$ at $95{ }^{\circ} \mathrm{C}, 35$ cycles of $30 \mathrm{~s}$ at $95^{\circ} \mathrm{C}, 30 \mathrm{~s}$ at $52-60{ }^{\circ} \mathrm{C}$, and $30 \mathrm{~s}$ at $72{ }^{\circ} \mathrm{C}$, and a final $7 \mathrm{~min}$ at $72{ }^{\circ} \mathrm{C}$. All the Sanger sequencing experiments were performed at Genomics (https://www.genomics.com.tw/, accessed on 24 December 2020).

\subsection{Whole Exome Sequencing and Data Analysis}

A total of 50 ng of DNA (based on Qubit quantification) were tagged and fragmented by a transposome and then cleaned up and amplified. A 200-400-bp band was selected, and exome capture was performed using the Nextera Exome Library Preparation Kit (Illumina, San Diego, CA, USA). The DNA library was quantified using a Qubit 3.0 Fluorometer (Life Technologies (Thermo Fisher Scientific), Waltham, MA, USA) and an Agilent 4200 Bioanalyzer (Agilent Technologies, Palo Alto, CA, USA). Samples were subjected to pairedend sequencing using the Illumina NovaSeq 6000 platform with a 150-bp read length. Details of the whole exome sequencing analysis have been described previously [38].

\subsection{Copy Number Variation Analysis}

DNA copy number profiling was performed using the customized Axiom-Taiwan Biobank Array (TWB chip: Affymetrix, Santa Clara, CA, USA). This customized array included 653,291 single nucleotide polymorphisms that were selected based specifically on the Taiwanese population, and genotyping was performed using standardized procedures of the National Center of Genome Medicine (Academia Sinica, Taipei, Taiwan). Axiom Analysis Suite (http:/ / www.affymetrix.com accessed on 7 January 2020) was used to call the copy number variation in each sample. The copy number variation was annotated using the AnnotSV tool [39].

\subsection{Statistical Analyses}

Clinicopathological samples were analyzed for the presence of fusion genes using Fisher's exact test. The Kaplan-Meier method was used to construct overall survival curves, which were then compared using the log-rank test. We compared variant numbers between patients with and without gene fusions using Welch's t-test. $p$-values $<0.05$ were considered to indicate significance. Statistical analyses were performed using SPSS v22. 


\section{Conclusions}

In this study, we identified known and novel fusion genes present in NSCLC patients using RNA-Seq and integrated gene expression, mutation, and fusion annotation data. Expression levels increased with ASXL1 (tumor suppressor gene), CACNA1A, EEF1A1, and RET (three oncogenes) fusions. The tumor grade and age were associated with fusion genes containing TSGs and tumor grade was also associated with fusion genes containing oncogenes. Nine patients carried both mutations and fusions of CDGs. Mutual exclusivity between the two types of genomic alteration, fusions and mutations, was observed. Furthermore, our study revealed an association between EGFR mutations and the number of fusion genes in the same patient. Some cancer patients may benefit from existing drugs that target fusion protein partners, expanding the therapeutic options available. Routine searches for fusion genes in cancer patients may improve their survival by revealing additional treatment targets for precise personalized medicine.

Supplementary Materials: The following are available online at https:/ / www.mdpi.com/2072-6 694/13/6/1343/s1, Figure S1: A novel fusion verified using RT-PCR and Sanger sequencing. The line represents the junction point of a fusion gene, Figure S2: Predictions of intrinsic disorder tendency of novel fusion proteins by IUPred2A (https: / /iupred2a.elte.hu/ (accessed on 3 November 2020)). Scores above 0.5 indicate disorder, Figure S3: Expression levels of genes present in fusions; expression without outliers are shown, Figure S4: Kaplan-Meier survival curves of patients with fusions containing TSGs and oncogenes, Figure S5: Schematic overview of oncogene mutation profiling, Table S1: Fusions found through RNA-Seq in 54 NSCLC patients, Table S2: Reported fusions identified in the Taiwanese cohort, Table S3: List of fusion numbers identified from 54 NSCLC patients, Table S4: Mutations in 299 cancer driver genes in 54 NSCLC patients, Table S5: Primer information for fusion gene validation.

Author Contributions: Conceptualization, J.-G.C.; Data curation, S.-J.T., J.-C.Y., and Y.-T.L.; Formal analysis, Y.-S.C.; Funding acquisition, J.-G.C.; Investigation, Y.-S.C.; Methodology, Y.-S.C., S.-J.T., J.-C.Y., and Y.-T.L.; Software, S.-J.T.; Supervision, J.-G.C.; Visualization, H.-Y.F.; Writing-review and editing, H.-Y.F., and J.-G.C. All authors have read and agreed to the published version of the manuscript.

Funding: This research was funded by China Medical University Hospital, grant number DMR-110-129.

Institutional Review Board Statement: The present study was approved by the Institutional Review Board of the China Medical University Hospital (CMUH106-REC1-053).

Informed Consent Statement: Informed consent was obtained from all subjects involved in the study.

Data Availability Statement: The RNA-Seq and WES data from this study were submitted to the NCBI Sequence Read Archive (SRA) under BioProject accession nos. PRJNA698419 and PRJNA698277, respectively.

Acknowledgments: The data analyzed in this study were partially provided by the China Medical University Hospital Cancer Registry

Conflicts of Interest: The authors declare no conflict of interest.

\section{References}

1. Siegel, R.L.; Miller, K.D.; Jemal, A. Cancer statistics, 2020. CA Cancer J. Clin. 2020, 70, 7-30. [CrossRef]

2. Bach, P.B. Smoking as a factor in causing lung cancer. JAMA 2009, 301, 539-541. [CrossRef] [PubMed]

3. Gould, M.K.; Silvestri, G.A.; Detterbeck, F. Multidisciplinary management of lung cancer. N. Engl. J. Med. 2004, 350, 2008-2010. [PubMed]

4. $\quad$ Ettinger, D.S.; Akerley, W.; Bepler, G.; Blum, M.G.; Chang, A.; Cheney, R.T.; Chirieac, L.R.; D’Amico, T.A.; Demmy, T.L.; Ganti, A.K.; et al. Non-small cell lung cancer. J. Natl. Compr. Canc. Netw. 2010, 8, 740-801. [CrossRef]

5. Zhang, X.C.; Wang, J.; Shao, G.G.; Wang, Q.; Qu, X.; Wang, B.; Moy, C.; Fan, Y.; Albertyn, Z.; Huang, X.; et al. Comprehensive genomic and immunological characterization of Chinese non-small cell lung cancer patients. Nat. Commun. 2019, 10, 1772. [CrossRef]

6. Ruiz-Cordero, R.; Devine, W.P. Targeted therapy and checkpoint immunotherapy in lung cancer. Surg. Pathol. Clin. 2020, 13, 17-33. [CrossRef] 
7. Gao, Q.; Liang, W.W.; Foltz, S.M.; Mutharasu, G.; Jayasinghe, R.G.; Cao, S.; Liao, W.W.; Reynolds, S.M.; Wyczalkowski, M.A.; Yao, L.; et al. Driver fusions and their implications in the development and treatment of human cancers. Cell Rep. 2018, 23, 227-238 e223. [CrossRef] [PubMed]

8. Aurias, A.; Rimbaut, C.; Buffe, D.; Dubousset, J.; Mazabraud, A. Translocation of chromosome 22 in Ewing's sarcoma. C. R. Seances Acad. Sci. III 1983, 296, 1105-1107. [PubMed]

9. Rowley, J.D. Letter: A new consistent chromosomal abnormality in chronic myelogenous leukaemia identified by quinacrine fluorescence and Giemsa staining. Nature 1973, 243, 290-293. [CrossRef] [PubMed]

10. Dinh, T.A.; Vitucci, E.C.; Wauthier, E.; Graham, R.P.; Pitman, W.A.; Oikawa, T.; Chen, M.; Silva, G.O.; Greene, K.G.; Torbenson, M.S.; et al. Comprehensive analysis of The Cancer Genome Atlas reveals a unique gene and non-coding RNA signature of fibrolamellar carcinoma. Sci. Rep. 2017, 7, 44653. [CrossRef]

11. Nikanjam, M.; Okamura, R.; Barkauskas, D.A.; Kurzrock, R. Targeting fusions for improved outcomes in oncology treatment. Cancer 2020, 126, 1315-1321. [CrossRef]

12. Lee, M.; Lee, K.; Yu, N.; Jang, I.; Choi, I.; Kim, P.; Jang, Y.E.; Kim, B.; Kim, S.; Lee, B.; et al. ChimerDB 3.0: An enhanced database for fusion genes from cancer transcriptome and literature data mining. Nucleic Acids Res. 2017, 45, D784-D789. [CrossRef]

13. Hu, X.; Wang, Q.; Tang, M.; Barthel, F.; Amin, S.; Yoshihara, K.; Lang, F.M.; Martinez-Ledesma, E.; Lee, S.H.; Zheng, S.; et al. TumorFusions: An integrative resource for cancer-associated transcript fusions. Nucleic Acids Res. 2018, 46, D1144-D1149. [CrossRef]

14. Stransky, N.; Cerami, E.; Schalm, S.; Kim, J.L.; Lengauer, C. The landscape of kinase fusions in cancer. Nat. Commun. 2014, 5, 4846. [CrossRef] [PubMed]

15. Rudin, C.M.; Durinck, S.; Stawiski, E.W.; Poirier, J.T.; Modrusan, Z.; Shames, D.S.; Bergbower, E.A.; Guan, Y.; Shin, J.; Guillory, J.; et al. Comprehensive genomic analysis identifies SOX2 as a frequently amplified gene in small-cell lung cancer. Nat. Genet. 2012, 44, 1111-1116. [CrossRef] [PubMed]

16. Seo, J.S.; Ju, Y.S.; Lee, W.C.; Shin, J.Y.; Lee, J.K.; Bleazard, T.; Lee, J.; Jung, Y.J.; Kim, J.O.; Shin, J.Y.; et al. The transcriptional landscape and mutational profile of lung adenocarcinoma. Genome Res. 2012, 22, 2109-2119. [CrossRef]

17. Kim, S.C.; Jung, Y.; Park, J.; Cho, S.; Seo, C.; Kim, J.; Kim, P.; Park, J.; Seo, J.; Kim, J.; et al. A high-dimensional, deep-sequencing study of lung adenocarcinoma in female never-smokers. PLOS ONE 2013, 8, e55596. [CrossRef] [PubMed]

18. Miyanaga, A.; Masuda, M.; Motoi, N.; Tsuta, K.; Nakamura, Y.; Nishijima, N.; Watanabe, S.I.; Asamura, H.; Tsuchida, A.; Seike, M.; et al. Whole-exome and RNA sequencing of pulmonary carcinoid reveals chromosomal rearrangements associated with recurrence. Lung Cancer 2020, 145, 85-94. [CrossRef] [PubMed]

19. Bailey, M.H.; Tokheim, C.; Porta-Pardo, E.; Sengupta, S.; Bertrand, D.; Weerasinghe, A.; Colaprico, A.; Wendl, M.C.; Kim, J.; Reardon, B.; et al. Comprehensive characterization of cancer driver genes and mutations. Cell 2018, 173, 371-385. [CrossRef]

20. Choi, Y.; Kwon, C.H.; Lee, S.J.; Park, J.; Shin, J.Y.; Park, D.Y. Integrative analysis of oncogenic fusion genes and their functional impact in colorectal cancer. Br. J. Cancer 2018, 119, 230-240. [CrossRef] [PubMed]

21. Manier, S.; Salem, K.Z.; Park, J.; Landau, D.A.; Getz, G.; Ghobrial, I.M. Genomic complexity of multiple myeloma and its clinical implications. Nat. Rev. Clin. Oncol. 2017, 14, 100-113. [CrossRef]

22. Lu, H.; Villafane, N.; Dogruluk, T.; Grzeskowiak, C.L.; Kong, K.; Tsang, Y.H.; Zagorodna, O.; Pantazi, A.; Yang, L.; Neill, N.J.; et al. Engineering and functional characterization of fusion genes identifies novel oncogenic drivers of cancer. Cancer Res. 2017, 77, 3502-3512. [CrossRef]

23. Shaw, A.T.; Kim, D.W.; Mehra, R.; Tan, D.S.; Felip, E.; Chow, L.Q.; Camidge, D.R.; Vansteenkiste, J.; Sharma, S.; De Pas, T.; et al. Ceritinib in ALK-rearranged non-small-cell lung cancer. N. Engl. J. Med. 2014, 370, 1189-1197. [CrossRef]

24. Deininger, M.W.; Druker, B.J. Specific targeted therapy of chronic myelogenous leukemia with imatinib. Pharmacol. Rev. 2003, 55, 401-423. [CrossRef] [PubMed]

25. Font-Tello, A.; Juanpere, N.; de Muga, S.; Lorenzo, M.; Lorente, J.A.; Fumado, L.; Serrano, L.; Serrano, S.; Lloreta, J.; Hernandez, S. Association of ERG and TMPRSS2-ERG with grade, stage, and prognosis of prostate cancer is dependent on their expression levels. Prostate 2015, 75, 1216-1226. [CrossRef] [PubMed]

26. Kurozumi, K.; Nakano, Y.; Ishida, J.; Tanaka, T.; Doi, M.; Hirato, J.; Yoshida, A.; Washio, K.; Shimada, A.; Kohno, T.; et al. Highgrade glioneuronal tumor with an ARHGEF2-NTRK1 fusion gene. Brain Tumor Pathol. 2019, 36, 121-128. [CrossRef] [PubMed]

27. Rosenkranz, A.A.; Slastnikova, T.A. Epidermal Growth Factor Receptor: Key to Selective Intracellular Delivery. Biochemistry 2020, 85, 967-1092. [CrossRef] [PubMed]

28. Bolger, A.M.; Lohse, M.; Usadel, B. Trimmomatic: A flexible trimmer for Illumina sequence data. Bioinformatics 2014, 30, 2114-2120. [CrossRef] [PubMed]

29. Chang, Y.S.; Tu, S.J.; Chiang, H.S.; Yen, J.C.; Lee, Y.T.; Fang, H.Y.; Chang, J.G. Genome-wide analysis of prognostic alternative splicing signature and splicing factors in lung adenocarcinoma. Genes 2020, 11, 1300. [CrossRef]

30. Kim, D.; Paggi, J.M.; Park, C.; Bennett, C.; Salzberg, S.L. Graph-based genome alignment and genotyping with HISAT2 and HISAT-genotype. Nat. Biotechnol. 2019, 37, 907-915. [CrossRef]

31. Liao, Y.; Smyth, G.K.; Shi, W. featureCounts: An efficient general purpose program for assigning sequence reads to genomic features. Bioinformatics 2014, 30, 923-930. [CrossRef]

32. Haas, B.J.; Dobin, A.; Li, B.; Stransky, N.; Pochet, N.; Regev, A. Accuracy assessment of fusion transcript detection via readmapping and de novo fusion transcript assembly-based methods. Genome Biol. 2019, 20, 213. [CrossRef] [PubMed] 
33. Yoshihara, K.; Wang, Q.; Torres-Garcia, W.; Zheng, S.; Vegesna, R.; Kim, H.; Verhaak, R.G. The landscape and therapeutic relevance of cancer-associated transcript fusions. Oncogene 2015, 34, 4845-4854. [CrossRef]

34. Klijn, C.; Durinck, S.; Stawiski, E.W.; Haverty, P.M.; Jiang, Z.; Liu, H.; Degenhardt, J.; Mayba, O.; Gnad, F.; Liu, J.; et al. A comprehensive transcriptional portrait of human cancer cell lines. Nat. Biotechnol. 2015, 33, 306-312. [CrossRef]

35. Meszaros, B.; Erdos, G.; Dosztanyi, Z. IUPred2A: Context-dependent prediction of protein disorder as a function of redox state and protein binding. Nucleic Acids Res. 2018, 46, W329-W337. [CrossRef]

36. Murphy, C.; Elemento, O. AGFusion: Annotate and visualize gene fusions. bioRxiv 2016. [CrossRef]

37. Sun, S.Q.; Mashl, R.J.; Sengupta, S.; Scott, A.D.; Wang, W.; Batra, P.; Wang, L.B.; Wyczalkowski, M.A.; Ding, L. Database of evidence for precision oncology portal. Bioinformatics 2018, 34, 4315-4317. [CrossRef] [PubMed]

38. Chang, C.C.; Chang, Y.S.; Huang, H.Y.; Yeh, K.T.; Liu, T.C.; Chang, J.G. Determination of the mutational landscape in Taiwanese patients with papillary thyroid cancer by whole-exome sequencing. Hum. Pathol. 2018, 78, 151-158. [CrossRef] [PubMed]

39. Geoffroy, V.; Herenger, Y.; Kress, A.; Stoetzel, C.; Piton, A.; Dollfus, H.; Muller, J. AnnotSV: An integrated tool for structural variations annotation. Bioinformatics 2018, 34, 3572-3574. [CrossRef] 